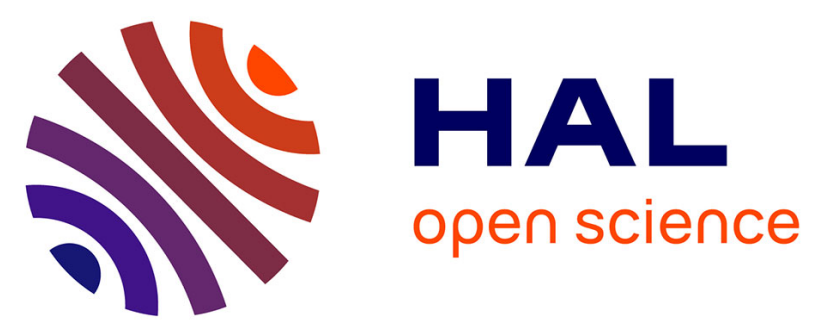

\title{
Fractal Energy Spectrum of a Polariton Gas in a Fibonacci Quasiperiodic Potential
}

\author{
Dimitrii Tanese, E. Gurevich, F. Baboux, T. Jacqmin, A. Lemaître, Elisabeth
} Galopin, Isabelle Sagnes, Alberto Amo, Jacqueline Bloch, E. Akkermans

\section{- To cite this version:}

Dimitrii Tanese, E. Gurevich, F. Baboux, T. Jacqmin, A. Lemaître, et al.. Fractal Energy Spectrum of a Polariton Gas in a Fibonacci Quasiperiodic Potential. Physical Review Letters, 2014, 112 (14), pp.146404. 10.1103/PhysRevLett.112.146404 . hal-03065613

\section{HAL Id: hal-03065613 https://hal.science/hal-03065613}

Submitted on 14 Dec 2020

HAL is a multi-disciplinary open access archive for the deposit and dissemination of scientific research documents, whether they are published or not. The documents may come from teaching and research institutions in France or abroad, or from public or private research centers.
L'archive ouverte pluridisciplinaire HAL, est destinée au dépôt et à la diffusion de documents scientifiques de niveau recherche, publiés ou non, émanant des établissements d'enseignement et de recherche français ou étrangers, des laboratoires publics ou privés. 


\title{
Fractal Energy Spectrum of a Polariton Gas in a Fibonacci Quasiperiodic Potential
}

\author{
D. Tanese, ${ }^{1}$ E. Gurevich, ${ }^{2}$ F. Baboux,${ }^{1}$ T. Jacqmin, ${ }^{1}$ A. Lemaitre, ${ }^{1}$ E. Galopin, ${ }^{1}$ I. Sagnes, ${ }^{1}$ A. Amo, ${ }^{1}$ \\ J. Bloch, ${ }^{1}$ and E. Akkermans ${ }^{2}$ \\ ${ }^{1}$ Laboratoire de Photonique et de Nanostructures, LPN/CNRS, Route de Nozay, 91460 Marcoussis, France \\ ${ }^{2}$ Department of Physics, Technion Israel Institute of Technology, Haifa 32000, Israel \\ (Received 11 November 2013; revised manuscript received 19 February 2014; published 10 April 2014)
}

\begin{abstract}
We report on the study of a polariton gas confined in a quasiperiodic one-dimensional cavity, described by a Fibonacci sequence. Imaging the polariton modes both in real and reciprocal space, we observe features characteristic of their fractal energy spectrum such as the opening of minigaps obeying the gap labeling theorem and log-periodic oscillations of the integrated density of states. These observations are accurately reproduced solving an effective 1D Schrödinger equation, illustrating the potential of cavity polaritons as a quantum simulator in complex topological geometries.
\end{abstract}

DOI: 10.1103/PhysRevLett.112.146404

PACS numbers: 71.36.+c, 61.43.Hv, 71.23.Ft, 78.67.-n

Free quantum particles or waves propagating in a spatially varying potential present modifications of their spectral density, which depend on the symmetry of this potential. The richness of spectral distributions in constrained geometries has long been recognized. The case of a periodic potential described by means of the Bloch theorem is a significant example. The notion of spectral distribution has been deepened in the wake of the discovery of quasicrystals and it led to a classification of energy spectra into absolutely continuous, pure point, and singular continuous spectral distributions [1]. The latter class proved to be surprisingly rich and it encompasses a broad range of potentials, such as quasiperiodic potentials which have been thoroughly studied $[2,3]$.

An interesting quasiperiodic potential can be designed using a Fibonacci sequence. The corresponding singular continuous energy spectrum has a fractal structure of the Cantor set type [4-7], and it displays self-similarity, i.e., a symmetry under a discrete scaling transformation. Denoting $\rho(\varepsilon)$ the relevant density of states (DOS) in $\varepsilon$ (either energy or frequency), a discrete scaling symmetry about a particular value $\varepsilon_{u}$ is expressed by the property

$$
\mu\left(\varepsilon_{u}+\Delta \varepsilon\right)-\mu\left(\varepsilon_{u}\right)=\frac{\mu\left(\varepsilon_{u}+\beta \Delta \varepsilon\right)-\mu\left(\varepsilon_{u}\right)}{\alpha},
$$

where $\mu(\varepsilon)=\int_{-\infty}^{\varepsilon} \rho\left(\varepsilon^{\prime}\right) d \varepsilon^{\prime}$ is the integrated density of states (IDOS), or density measure, and $\alpha$ and $\beta$ are scaling parameters which usually, depend on $\varepsilon_{u}$. Defining a shifted IDOS by $\mathcal{N}_{\varepsilon_{u}}(\varepsilon) \equiv \mu(\varepsilon)-\mu\left(\varepsilon_{u}\right)$, the general solution of Eq. (1) can be written as [8]

$$
\mathcal{N}_{\varepsilon_{u}}(\varepsilon)=\left|\varepsilon-\varepsilon_{u}\right|^{\gamma} \mathcal{F}\left(\frac{\ln \left|\varepsilon-\varepsilon_{u}\right|}{\ln \beta}\right)
$$

where $\gamma=\ln \alpha / \ln \beta$ is the local ( $\varepsilon_{u}$-dependent) scaling exponent and $\mathcal{F}(z)$ is a periodic function of period unity, whose (nonuniversal) form depends on the problem at hand. Generally, the exponent $\gamma$ takes values between zero and unity, so that the density $\rho(\varepsilon)$ is a singular function. Such scaling properties of a fractal spectrum are expected to modify the behavior of physical quantities [8]. Recently studied examples include thermodynamic properties of photons [9], random walks [10], the quantum diffusion of wave packets [11], and spontaneous emission triggered by a fractal vacuum [12]. The diffusion of a wave packet in a quasiperiodic medium is predicted to be neither diffusive, nor ballistic but to present a behavior characterized by nonuniversal exponents and a log-periodic modulation of its time dynamics. Experimental demonstration of these specific properties of quasiperiodic structures is still missing as yet. We propose to use cavity polaritons to evidence such a fractal behavior.

Cavity polaritons are quasiparticles arising from the strong coupling between the optical mode of an optical cavity and excitons confined in quantum wells [13]. They have appeared recently as a promising system to realize quantum simulators [14,15]. Engineering of the potential landscape is possible and allows implementing a large variety of physical situations such as 1D [14,16,17] and 2D periodic potentials $[18,19]$ with the generation of gap solitons [17,20], nonlinear resonant tunneling devices [21], or triangular [22] and honeycomb [23,24] lattices, which enables the exploration of graphene physics. Polaritons offer experimental possibilities not available in $1 \mathrm{D}$ or $2 \mathrm{D}$ photonic quasicrystals such as direct timeand energy-resolved measurements of the excitations in both space and momentum domains. Thus, one can directly visualize individual eigenmodes, and the dynamics of wave packets.

In this Letter, we use this well-controlled system to investigate both theoretically and experimentally the spectral properties of a polariton gas in a quasiperiodic potential. To do so, we have sculpted the lateral profile 

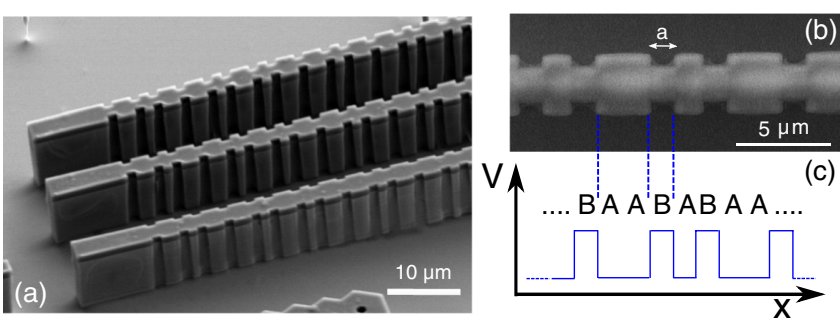

FIG. 1 (color online). (a) Scanning electron microscopy image of an array of modulated wires. (b) Zoom on a particular wire, showing the shape of the $A$ and $B$ letters. (c) Schematic of the nominal potential corresponding to the lateral shaping of the wire cavity.

of a quasi-1D cavity in the shape of a Fibonacci sequence. Using nonresonant excitation in the low density regime, we probe the modes both in real and reciprocal space. We observe a quantitative agreement between experiments and the calculated modes and density of states. In particular, we evidence features of a fractal energy spectrum, namely gaps densely distributed and an integrated density of states reflecting the existence of a discrete scaling symmetry as expressed by Eq. (2).

In our sample, cavity polaritons are confined within narrow strips (wire cavities), whose width is modulated quasiperiodically. These wires are fabricated by processing a planar high quality factor $(Q \sim 72000)$ microcavity grown by molecular beam epitaxy. It consists in a $\lambda / 2$ $\mathrm{Ga}_{0.05} \mathrm{Al}_{0.95} \mathrm{As}$ layer surrounded by two $\mathrm{Ga}_{0.8} \mathrm{Al}_{0.2} \mathrm{As}$ / $\mathrm{Ga}_{0.05} \mathrm{Al}_{0.95} \mathrm{As}$ Bragg mirrors with 28 and 40 pairs in the top/bottom mirrors, respectively. Twelve GaAs quantum wells of width $7 \mathrm{~nm}$ are inserted in the structure resulting in a $15 \mathrm{meV}$ Rabi splitting. $200 \mu \mathrm{m}$ long wires with the lateral dimension modulated quasiperiodically are designed using electron beam lithography and dry etching [Figs. 1(a) and 1(b)]. The modulation consists in two wire sections ("letters") $A$ and $B$ of same length $a$ but different widths $w_{A}$ and $w_{B}$ respectively [Fig. 1(b)]. The modulation of the wire width induces an effective 1D potential for the longitudinal motion of polaritons, as discussed in the sequel. The letters are arranged according to the Fibonacci sequence [4] using the recursion,

$$
S_{j \geq 3}=\left[S_{j-2} S_{j-1}\right], \quad \text { and } \quad S_{1}=B, \quad S_{2}=A,
$$

where $\left[S_{j-2} S_{j-1}\right]$ means concatenation of two subsequences $S_{j-2}$ and $S_{j-1}$. The number of letters (length) of a sequence $S_{j}$ is given by the Fibonacci number $F_{j}$, such that $F_{j+1}=F_{j}+F_{j-1}$. The ratio $F_{j+1} / F_{j}$ tends to the golden mean $\sigma=(1+\sqrt{5}) / 2 \simeq 1.62$ in the limit $j \rightarrow \infty$, while the corresponding sequence $S_{\infty}$ becomes rigorously quasiperiodic and invariant, i.e., self-similar, under the iteration transformation Eq. (3). Our sample corresponds to $S_{13}$ counting 233 letters with $a=0.8 \mu \mathrm{m}, w_{A}=3.5 \mu \mathrm{m}$ and $w_{B}=1.86 \mu \mathrm{m}$. To study the polariton modes in these

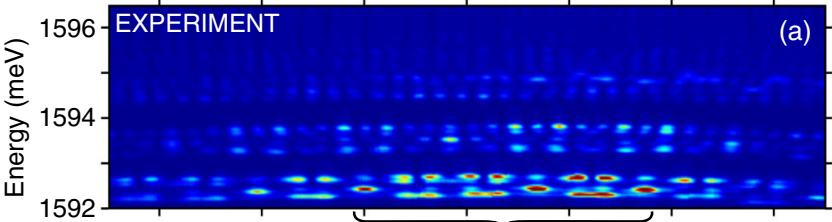

$S_{13}=\ldots$ AaBABAABAABABAABAABABAABABAABAABABAA$\ldots$

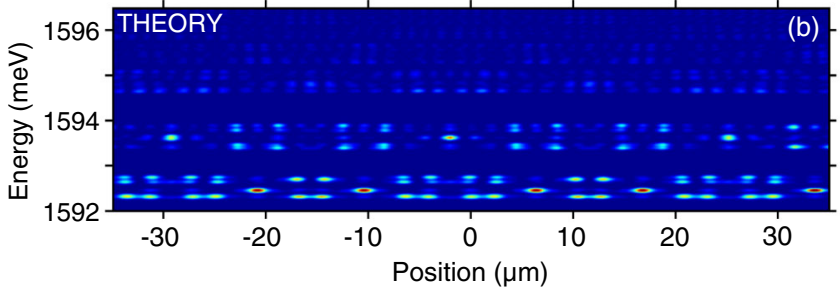

FIG. 2 (color online). (a) Spectrally and spatially resolved emission measured on a single modulated wire (the linear polarization parallel to the wire is selected). Bottom of the figure: letter sequence corresponding to a part of the whole $S_{13}$ potential sequence. (b) Calculated polariton Fibonacci modes as a function of energy and real space coordinate.

quasiperiodic wires, we perform low temperature $(10 \mathrm{~K})$ microphotoluminescence experiments. Single wires are excited nonresonantly using a cw monomode laser tuned typically $100 \mathrm{meV}$ above the polariton resonances. The excitation spot extends over a $80 \mu \mathrm{m}$-long region along the wire. The sample emission is collected with a 0.65 numerical aperture objective and focused on the entrance slit (parallel to the wire) of a spectrometer coupled to a CCD camera. Imaging of the sample surface (the Fourier plane of the collection objective) allows for studying the spectrally resolved polariton modes in real (reciprocal) space. Excitation power is kept low enough to stay below the condensation threshold and obtain a nearly homogeneous population of the lower energy polariton states.

Figure 2(a) displays the spatially and spectrally resolved emission measured on a single modulated wire cavity for an exciton-photon detuning around $-8 \mathrm{meV}$ (defined as the energy difference between the cavity mode at normal incidence and the exciton resonance). Several polariton modes are imaged. They present complex patterns of bright spots distributed all over the region of the wire under investigation. To understand the nature of these modes and properties of their spectral density, we have calculated the polariton eigenstates in such quasiperiodic structures.

In our model, whose details are given in the Supplemental Material [25], we describe the confined photon modes using a 2D scalar wave equation with vanishing boundary conditions on the boundary of the wire, considered as an axially symmetric strip where the longitudinal coordinate $x \in[0, L]$ ( $L$ being the length of the wire), and the transverse coordinate $-w(x) / 2 \leq y \leq w(x) / 2$. Here, $w(x)>0$ accounts for the $x$-dependent width of the wire [Fig. 1(c)], i.e., a quasiperiodic sequence of segments of width $w_{A}$ and $w_{B}$, as defined in Eq. (3). In the Supplemental Material [25], we 
show how to map this 2D problem onto a 1D Schrödinger equation with the effective potential:

$$
V(x)=\frac{\pi^{2}}{w^{2}(x)}+\frac{\pi^{2}+3}{12}\left(\frac{w^{\prime}(x)}{w(x)}\right)^{2} .
$$

The first term of $V(x)$ is the usual adiabatic approximation. The second term accounts for the sharpness of the steps. It is not perturbative, and it cannot be neglected (see Supplemental Material [25]). As clearly visible in Fig. 1, the strip shape is not perfectly abrupt but presents some smoothness in the width variation introduced by the actual etching process. The smoothness scale is used as a fitting parameter in the calculations. The eigenfunctions $\phi_{q}(x)$ and eigenenergies $E_{C, q}$ are obtained numerically. To calculate the polariton modes, we consider the radiative coupling between excitons with a flat dispersion to the photon modes which we have obtained in our simulations. Since the coupling is diagonal in the index $q$, the resulting polariton eigenfunctions and photons have the same spatial behavior. Figure 2(b) shows the polariton modes thus obtained numerically. Since experimentally we cannot resolve states which are separated by less than the polariton linewidth, we have averaged the intensity over eigenmodes close in energy. Thus, what appears in Fig. 2(b) as bright intensity spots at different energies are actually bands separated by gaps. Clearly the calculation reproduces very accurately the spatial structure of the polariton modes observed in the experiment. This direct imaging of the Fibonacci modes in a quasiperiodic structure is a clear asset offered by cavity polaritons.

Probing the polariton modes in reciprocal space also provides remarkable information about the eigenmodes. This is illustrated on Fig. 3(a), where taking advantage of the one-to-one relation between the angle of emission and in-plane momentum of polaritons, far field imaging of the polariton emission is shown for the same wire as in Fig. 2. A complex band structure appears with the opening of gaps not regularly spaced unlike the case of a periodic modulation [17]. The calculated band structure reproduces the measurements quantitatively [Fig. 3(b)].

In the rest of the Letter, we show that despite the finite size of the system, both in the numerics and in the experiments, fundamental physical properties are evidenced in this complex band structure which indicate the onset of a fractal density of states. To study the spectrum and the position of its gaps, it is convenient to rewrite the quasiperiodic potential $V(x)$ in Eq. (4) under the form,

$$
V(x)=\sum_{n} \chi\left(\sigma^{-1} n\right) u_{b}(x-a n)
$$

valid in principle [4] for an infinitely long system namely $j \rightarrow \infty$ in (3). $u_{b}(x)$ [which depends on $w(x)$ ] describes the shape of the letter $B$ while the periodic function $\chi(x)$ defined, within $[0,1]$, by $\chi(x)=1$ for $0<x<2-\sigma$ and
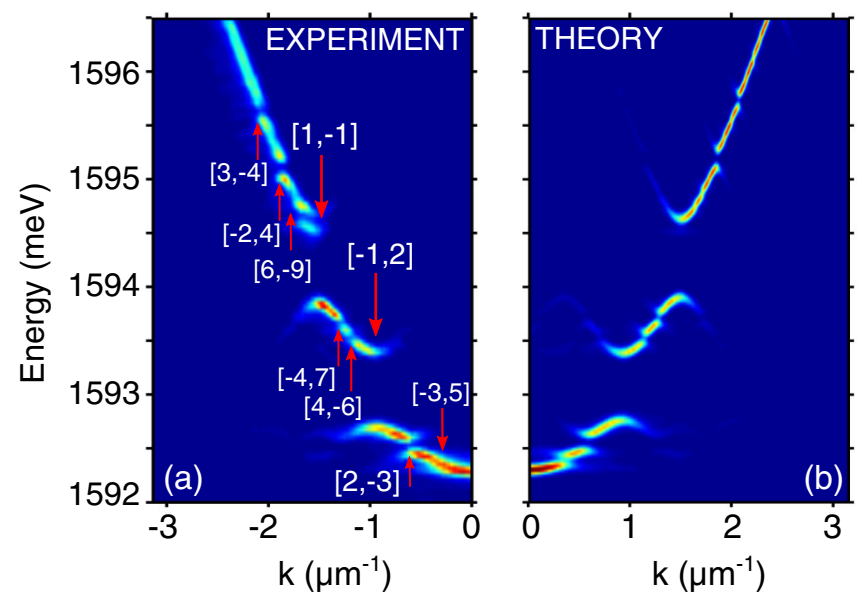

FIG. 3 (color online). (a) Spectrally resolved far field emission measured on the same wire cavity used in Fig. 2. (b) Corresponding simulation. Position of the gaps labeled with two integers $[p, q]$ is indicated with red arrows.

$\chi(x)=0$ for $2-\sigma<x<1$, accounts for the quasiperiodic order. The Fourier transform of $V(x)$ consists of Bragg peaks and is given by

$$
V(k)=\tilde{u}_{b}(k) \sum_{p, q} \chi_{q} \delta\left(k a-2 \pi\left(p+q \sigma^{-1}\right)\right)
$$

in standard notation. Since $\sigma$ is irrational, each Bragg peak of the quasiperiodic potential can be uniquely labeled with a set $[p, q]$ of two integers so that the corresponding wave number is $k=Q_{p, q} \equiv(2 \pi / a)\left(p+q \sigma^{-1}\right)$. Similarly to the Bloch theorem for a periodic modulation, we may expect that a series of gaps opens at each independent Bragg peak $Q_{p, q}$. Thus, to label the gaps and to obtain the IDOS given in Eq. (2), it is tempting to consider the quasiperiodic potential $V(x)$ as a small perturbation. Albeit not justified in the present experimental case, we shall first use this assumption since it allows us to give a more intuitive derivation of the gap labeling. But the Bragg peaks being a dense set, we must be cautious and first approximate $\sigma$ by its finite approximants $\sigma_{j}=F_{j+1} / F_{j}$ as defined after Eq. (3). Then, $V(x)$ in Eq. (5) becomes a periodic approximant $V_{j+1}(x)$, built from periodically repeated cells $S_{j+1}$ of length $a F_{j+1}$. Thus, the properties of the single cell $S_{j+1}$ studied experimentally are essentially those of the periodic potential $V_{j+1}(x)$. Its Fourier transform $V_{j+1}(k)$ is obtained replacing $\sigma$ by $\sigma_{j}$ in Eq. (6). $V_{j+1}(k)$ thus defined, is the structure factor of a periodic structure and therefore it has a finite density of Bragg peaks spaced by $\Delta k=2 \pi /\left(a F_{j+1}\right)$. Perturbation theory in $|V| \ll 1$ is now applicable. To first order, each Bragg peak $k=Q_{p, q} \equiv$ $(2 \pi / a)\left(F_{j+1} p+F_{j} q\right)$ hybridizes the degenerate Bloch waves at wave numbers $\pm Q_{p, q} / 2$. The coupling between these plane waves is best described by a two-level 

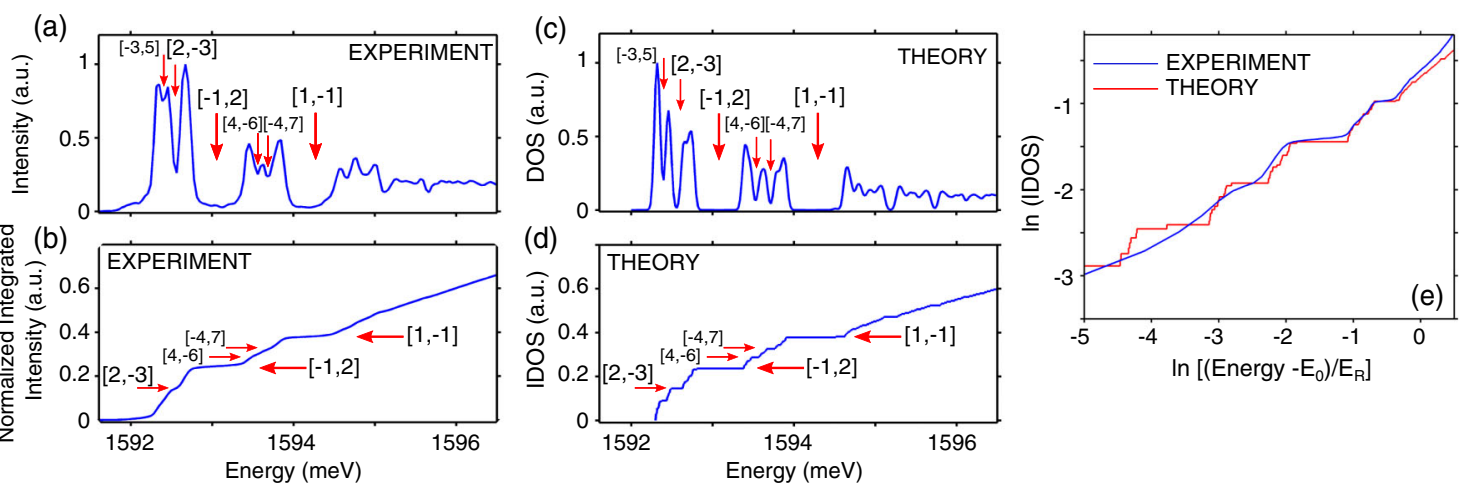

FIG. 4 (color online). (a) Measured total (angularly integrated) emission spectrum $I(\varepsilon)$ of the quasiperiodic wire and (b) spectrally integrated emission intensity $\int_{E_{0}}^{\varepsilon} I\left(\varepsilon^{\prime}\right) d \varepsilon^{\prime}$ (where $E_{0}$ is the lower energy state). Calculated normalized DOS (c) and IDOS (d). (e) Display of the log-periodic oscillations of the IDOS in a log-log plot of numerical (red) and experimental (blue) IDOS in the vicinity of $E_{0}$ (normalized to $E_{R}=\hbar^{2} \pi^{2} /\left(8 a^{2} m_{p}\right)$, with $m_{p}$ the polariton mass).

Hamiltonian with diagonal, $\varepsilon \equiv E_{Q_{p, q} / 2}=E_{-Q_{p, q} / 2}$, and off-diagonal, $V_{q} \equiv V \chi_{q}$, matrix elements. The doubly degenerate level $\varepsilon$ splits into $\varepsilon \pm\left|V_{q}\right|$ and a gap of width $2\left|V_{q}\right|$ opens at this energy. Accordingly, there is a one-toone correspondence between the Bragg peaks and the gaps generated through the hybridization of plane waves, so that each gap can also be labeled with the two integers $[p, q]$. Noting that $Q_{p, q} a / 2 \pi=p+q \sigma^{-1}$ is the proportion of unperturbed eigenmodes whose energies are less than $\varepsilon=E_{Q_{p, q} / 2}$, the IDOS inside the $[p, q]$-gap is

$$
\mathcal{N}\left(\varepsilon=E_{Q_{p, q} / 2}\right)=p+q \sigma^{-1}=q \sigma^{-1}(\bmod .1)
$$

for $\mathcal{N}\left(\varepsilon=E_{Q_{p, q} / 2}\right)$ normalized to unity at $E_{Q_{1,0}}$.

While the previous result has been obtained using perturbation theory, it happens that it has a much broader range of validity generally expressed by the so-called gap labeling theorem [28] formulated by Bellissard and coworkers. This theorem provides a precise framework for applicability and allows us to compute values of the IDOS in the gaps of the spectrum of 1D Schrödinger Hamiltonians with bounded potentials $V(x)$. An important consequence of that theorem is the topologically stable nature of the IDOS values in the gaps which extends beyond perturbation theory. Those specific values are obtained [28] from some prescribed linear combinations of components of eigenvectors of the corresponding substitution matrix characteristic of the quasiperiodic potential. For the Fibonacci sequence defined in Eq. (3), that prescription reduces to linear combinations of 1 and $\sigma^{-1}$, namely, to Eq. (7). In Fig. 3(a), we indicate with red arrows the labeling of the gaps using the set $[p, q]$, demonstrating that the positions of the gaps are accurately determined by the positions of the Bragg peaks even for a relatively short Fibonacci sequence such as considered here. These positions are topological quantities, namely, independent of the strength of the potential. These observed spectral features are thus independent of the (large enough) sample size and of the realization of the potential. These points are further discussed in the Supplemental Material [25]. On the other hand, the energy width of the gaps depends on the heights of the Bragg peaks, i.e., on the details of the potential $u_{b}(x)$ [and $w(x)$ ].

The peculiar structure of the emission spectrum appears also clearly by considering the total emission intensity $I(\varepsilon)$ nearly proportional to the DOS for low excitation powers. Figure 4(a) displays peaks and dips corresponding, respectively, to bands and pseudogaps. The measured integrated intensity $\int_{E_{0}}^{\varepsilon} I\left(\varepsilon^{\prime}\right) d \varepsilon^{\prime}$ (with $E_{0}$ being the lower energy state), is reported in Fig. 4(b) together with the numerically calculated DOS and IDOS [Figs. 4(c) and 4(d)]. Applying Eq. (7), valid in principle in the infinite limit, to the gaps $[2,-3],[-1,2],[1,-1]$ indicated in Figs. 4(b)-(d), gives, respectively, $\mathcal{N}\left(E_{Q_{p, q} / 2}\right)=0.15,0.24,0.38$. These numbers are in excellent agreement with the experiment, confirming the good homogeneity achieved in populating the polariton states.

For the infinite system, there exists an infinite series of gaps at $p+q \sigma^{-1} \in[0,1]$. Thus the energy spectrum, which is the complementary of these gaps, is singular continuous. It is a Cantor-like set whose total width vanishes. The high resolution available in the numerics allows us to consider finer details of the IDOS as predicted by the scaling form Eq. (2). In Fig. 4(e), we have plotted in a log-log scale the IDOS as a function of (properly normalized) energy. It is noticeable that, even for such a finite sized system, we indeed observe a power law behavior multiplied by a logperiodic function. More interesting is the experimental observation of these log-periodic oscillations, showing two periods of oscillations, which constitutes a direct and so far unobserved signature of the fractal character of the Fibonacci spectrum.

In summary, probing the luminescence of a polariton gas laterally confined by a Fibonacci quasiperiodic potential, we have observed the characteristic behavior of the associated fractal energy spectrum: gaps densely distributed, and an IDOS well described by the scaling form 
Eq. (2) and following the gap labeling theorem Eq. (7). We have obtained a spectrally and spatially resolved image of the polariton modes which is in good quantitative agreement with theoretical and numerical results. Our results support the idea that topological features of a fractal spectrum are robust and show up quite accurately even for a relatively short structure. Those results evidence the great interest of cavity polaritons to study the anomalous time expansion of a polariton wave packet [11], more complex quantum systems, e.g., 2D quasicrystals [29], and more generally to realize quantum simulators.

This work was supported by the Israel Science Foundation Grant No. 924/09, by the "Agence Nationale pour la Recherche" project "Quandyde" (ANR-11-BS10001), by the FP7 ITN "Clermont4" (235114), by the French RENATECH network, the LABEX NanoSaclay and the Honeypol ERC starting grant.

[1] H. L. Cycon, R. G. Froese, W. Kitsch, and B. Simon, Schrödinger Operators (Springer, Berlin, 1987); M. Reed and B. Simon, Methods of Modern Mathematical Physics (Academic Press, California, 1980).

[2] D. Damanik and A. Gorodetski, Commun. Math. Phys. 305, 221 (2011); D. Damanik, M. Embree, A. Gorodetski, and S. Tcheremchantsev, Commun. Math. Phys. 280, 499 (2008).

[3] For a recent review see Z. V. Vardeny, A. Nahat, and A. Agrawal, Nat. Photonics 7, 177 (2013).

[4] M. Kohmoto, B. Sutherland, and C. Tang, Phys. Rev. B 35, 1020 (1987); J. M. Luck, Phys. Rev. B 39, 5834 (1989).

[5] W. Gellermann, M. Kohmoto, B. Sutherland, and P. C. Taylor, Phys. Rev. Lett. 72, 633 (1994).

[6] M. Kohmoto, L. P. Kadanof, and C. Tang, Phys. Rev. Lett. 50, 1870 (1983); S. Ostlund and S. Kim, Phys. Scr. T9, 193 (1985); For a review see E. L. Albuquerque and M. G. Cottam, Phys. Rep. 376, 225 (2003); E. Maciá, Rep. Prog. Phys. 69, 397 (2006).

[7] M. Kohmoto, B. Sutherland, and K. Iguchi, Phys. Rev. Lett. 58, 2436 (1987); L. DalNegro, C. J. Oton, Z. Gaburro, L. Pavesi, P. Johnson, A. Lagendijk, R. Righini, M. Colocci, and D. S. Wiersma, Phys. Rev. Lett. 90, 055501 (2003); D. Würtz, T. Schneider, and M. P. Soerensen, Physica (Amsterdam) 148A, 343 (1988).

[8] For a recent review, E. Akkermans, Contemp. Math. 601, 1 (2013).

[9] E. Akkermans, G. V. Dunne, and A. Teplyaev, Phys. Rev. Lett. 105, 230407 (2010).

[10] E. Akkermans, O. Benichou, G. V. Dunne, A. Teplyaev, and R. Voituriez, Phys. Rev. E 86, 061125 (2012).

[11] I. Guarneri and G. Mantica, Phys. Rev. Lett. 73, 3379 (1994); S. Abe and H. Hiramoto, Phys. Rev. A 36, 5349 (1987).
[12] E. Akkermans and E. Gurevich, Europhys. Lett. 103, 30009 (2013).

[13] C. Weisbuch, M. Nishioka, A. Ishikawa, and Y. Arakawa, Phys. Rev. Lett. 69, 3314 (1992).

[14] C. W. Lai, N. Y. Kim, S. Utsunomiya, G. Roumpos, H. Deng, M. D. Fraser, T. Byrnes, P. Recher, N. Kumada, T. Fujisawa, and Y. Yamamoto, Nature (London) 450, 529 (2007).

[15] I. Carusotto and C. Ciuti, Rev. Mod. Phys. 85, 299 (2013).

[16] E. A. Cerda-Méndez, D. N. Krizhanovskii, M. Wouters, R. Bradley, K. Biermann, K. Guda, R. Hey, P. V. Santos, D. Sarkar, and M. S. Skolnick, Phys. Rev. Lett. 105, 116402 (2010).

[17] D. Tanese, H. Flayac, D. Solnyshkov, A. Amo, A. Lemaître, E. Galopin, R. Braive, P. Senellart, I. Sagnes, G. Malpuech, and J. Bloch, Nat. Commun. 4, 1749 (2013).

[18] N. Y. Kim, K. Kusudo, C. Wu, N. Masumoto, A. Löffler, S. Höfling, N. Kumada, L. Worschech, A. Forchel and Y. Yamamoto, Nat. Phys. 7, 681 (2011).

[19] E. A. Cerda-Méndez, D. N. Krizhanovskii, K. Biermann, R. Hey, M. S. Skolnick, and P. V. Santos, Phys. Rev. B 86, 100301 (2012).

[20] E. A. Cerda-Méndez, D. Sarkar, D. N. Krizhanovskii, S. S. Gavrilov, K. Biermann, M. S. Skolnick, and P. V. Santos, Phys. Rev. Lett. 111, 146401 (2013).

[21] H. S. Nguyen, D. Vishnevsky, C. Sturm, D. Tanese, D. Solnyshkov, E. Galopin, A. Lemaître, I. Sagnes, A. Amo, G. Malpuech, and J. Bloch, Phys. Rev. Lett. 110, 236601 (2013).

[22] N. Y. Kim, K. Kusudo, A. Löffler, S. Höfling, A. Forchel, and Y. Yamamoto, New J. Phys. 15, 035032 (2013).

[23] K. Kusudo, N. Y. Kim, A. Löffler, S. Höfling, A. Forchel, and Y. Yamamoto, Phys. Rev. B 87, 214503 (2013).

[24] T. Jacqmin, I. Carusotto, I. Sagnes, M. Abbarchi, D. Solnyshkov, G. Malpuech, E. Galopin, A. Lemaître, J. Bloch, and A. Amo, Phys. Rev. Lett. 112, 116402 (2014).

[25] See Supplemental Material at http://link.aps.org/ supplemental/10.1103/PhysRevLett.112.146404 that includes Refs. [26,27], for additional data on wires with different structural parameters and for an explicit derivation of Eq. (4) and a discussion of the validity of the effective 1D potential.

[26] A. Kuther, M. Bayer, T. Gutbrod, A. Forchel, P. A. Knipp, T. L. Reinecke, and R. Werner, Phys. Rev. B 58, 15744 (1998).

[27] S. Schwartz, M. Cozzini, C. Menotti, I. Carusotto, P. Bouyer and S. Stringari, New J. Phys. 8, 162 (2006).

[28] J. Bellissard, A. Bovier, and J. M. Ghez, Rev. Math. Phys. 04, 1 (1992); B. Simon, Adv. Appl. Math. 3, 463 (1982); J. Bellissard, in "Number Theory and Physics," Proceedings of Les Houches, edited by J. M. Luck, P. Moussa, and M. Waldschmidt (Springer, New York, 1993).

[29] J.-M. Gambaudo and P. Vignolo, arXiv:1309.6420 [New J. Phys. (to be published)]. 